\title{
A Possible Accelerator Experiment to Solve the Solar-Neutrino
}

\section{Puzzle.}

A. CAPONE and H. WACHSMUTH

CERN - Geneva

\section{Palladino}

Dipartimento di Fisica dell'Università - Napoli, Italia Isitutó Nazionale di Fisica Nucleare - Sezione di Napoli, Italia

M. De Vincenzi, U. Dore, O. Palamara, P. Pistilli and D. Zanello Dipartimento di Fisica dell'Università - Roma, Italia Isituto Nazionale di Fisica Nucleare - Sezione di Roma, Italia

(ricevuto il 29 Aprile 1987)

Summary. - We show the possibility to use an intense neutrino beam from an accelerator combined with an underground detector to study the $v$ oscillation. In particular, we discuss the configurations in which the sensitivity to oscillation parameters is comparable with solar-neutrino experiments.

PACS. 13.15. - Neutrino interactions (including reactions involving cosmic raýs).

\section{1. - Introduction.}

.

In order to make substantial improvements on the limits on y oscillation parameters one needs long-distance, high-intensity $y$ beams and large-mass detectors.

The idea of using an underground detector placed in the Gran Sasso Laboratory and to steer into it $y$ beams generated at CERN has been put forward long time ago $\left(^{1}\right)$.

(1) A. ZICHICHI: The Gran Sasso project (Gud Workshop, Rome, 1981). 
In this paper we show that; if one uses a high-intensity $30 \mathrm{GeV}$ proton beam as proposed by the European Hadron Facility (EHF) $\left({ }^{2}\right)$ and the ICARUS detector $\left(^{3}\right)$, one can reach a sensitivity to the oscillation parameters comparable to that of the solar-neutrino experiments.

In fact, it may turn out that the comparison of the result of the two kinds of experiments be crucial in order to distinguish between the hypothesis of the " oscillation or the breakdown of the standard solar model.

In the first part of this paper the problems related to solar neutrinos will be reviewed briefly; in the second part the experimental scheme will be discussed.

\section{2. - Solar neutrinos.}

The energy produced in the Sun is released in the processes based on the mechanism of fusion of light elements into heavier ones starting with atomic hydrogen. The most important reactions believed to produce solar neutrinos with continuous energy spectra are $\left(^{4}\right)$

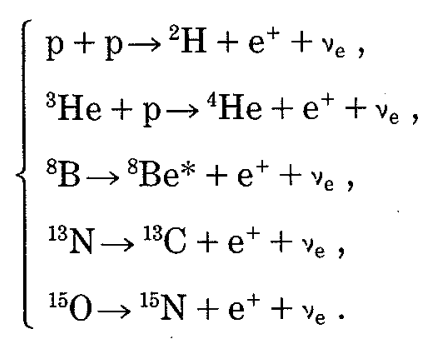

Moreover, electron capture reactions produce almost monochromatic neutrinos. The most important neutrino lines come from the processes

$$
\mathrm{p}+\mathrm{p}+\mathrm{e}^{-} \rightarrow{ }^{2} \mathrm{H}+v_{\mathrm{e}}, \quad{ }^{7} \mathrm{Be}+\mathrm{e}^{-} \rightarrow{ }^{7} \mathrm{Li}+\nu_{\mathrm{e}} .
$$

:

These processes give rise to the neutrino energy spectrum computed in the standard solar model shown in fig. 1.

The only experiment performed up to now to detect solar neutrinos is that of Davis et al. $\left(^{(5)}\right.$ who used the reaction

$$
{ }_{\mathrm{e}}+{ }^{37} \mathrm{Cl} \rightarrow{ }^{37} \mathrm{Ar}+\mathrm{e}^{-}
$$

$\left({ }^{2}\right)$ EHF letter of intent, Mainz, August, 1986.

(3) ICARUS proposal, INFN/AE-85/7.

(') J. N. BahCall: Rev. Mod. Phys., 50, 881 (1978).

(5) R. Davis, B. T. Cleveland, J. C. Evans and J. K. Rowley: Bull. Amer. Phys. Soc. (April 1984), p. 731; R. Davis, B. T. Cleveland and J. K. Rowley: AIP Conference Proceedings (Los Alamos, 1982). 


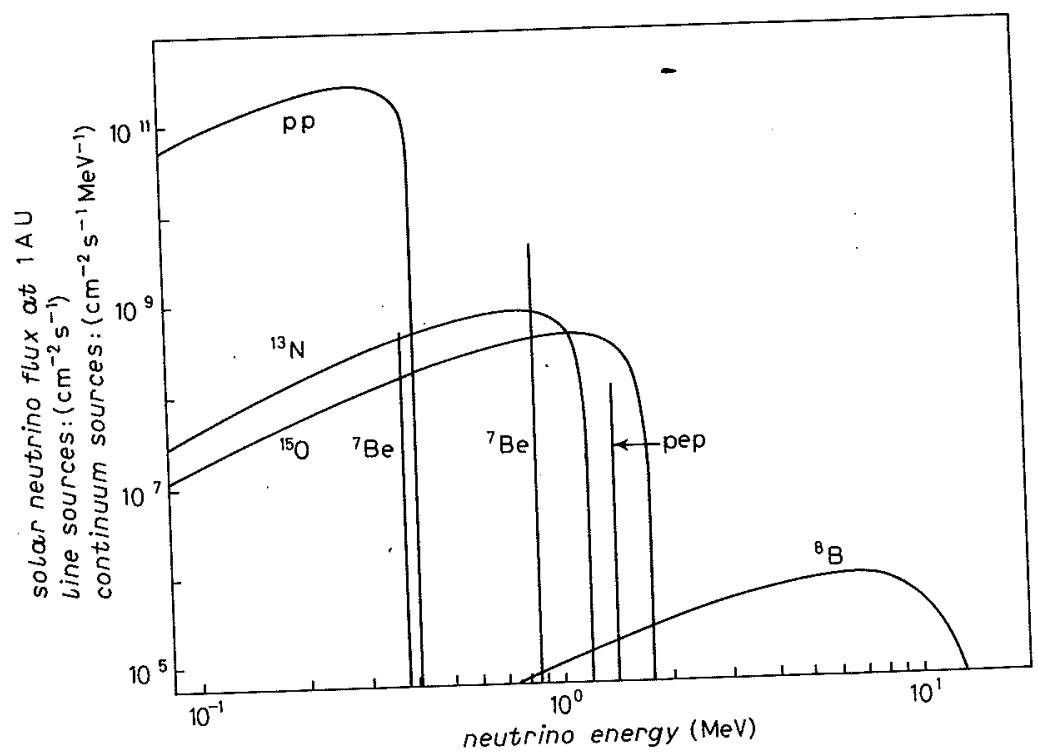

Fig. 1. - Neutrino energy spectrum as computed in the standard solar model.

with an energy threshold of $814 \mathrm{keV}$. Taking into account the energy behaviour of the cross-section for reaction (3), the experiment of Davis turns out to be mainly sensitive to the high-energy tail $(E>2 \mathrm{MeV})$ of the solar-neutrino spectrum coming from the ${ }^{8} \mathrm{~B}$ decay. The result of the experiment is the measured rate of $(2.1 \pm 0.3) \cdot 10^{36} v_{e}$ captures/s target nucleus, this rate is about three times smaller than expected from the standard solar model. This result can be attributed either to a fault of the standard solar model, or to a fault of the nuclear-physics calculations of the cross-section of reactions (1), or to neutrino oscillations.

In fact, considering for the sake of simplicity two neutrinos only, if the weakinteraction eigenstates $\left(v_{e}, v_{4}\right)$ do not coincide with the mass eigenstates $\left(v_{1}, v_{2}\right)$ one has

$$
v_{\mathrm{e}}=v_{1} \cos \theta+v_{2} \sin \theta, \quad v_{\mu}=-v_{1} \sin \theta+v_{2} \cos \theta
$$

The probability to ind a $v_{\mu}$ starting from a pure $v_{\mathrm{e}}$ state at a distance $L$ (in $\mathrm{km}$ ) from the source is given by

$$
P\left(v_{\mathrm{e}} \rightarrow v_{\mu}\right)=\sin ^{2} 2 \theta \cdot \sin ^{2}\left(1.27 \Delta m^{2} \frac{L}{E}\right)
$$

where $\Delta m^{2}=m_{1}^{2}-m_{2}^{2}\left(m_{1}\right.$ and $m_{2}$, mass eigenvalues in $\left.\mathrm{eV}\right)$ and $E$ is the neutrino energy in GeV. Since the $v_{\mu}$ cannot activate reaction (3), the mechanism of the neutrino oscillation gives a reduction of the $y_{e}$ flux coming from the Sutn as 
measured on the Earth. Moreover, Mikheyev and Smirnov have recently observed that, due to the so-called matter effect $\left(^{6}\right)$ in the Sun, the oscillation between neutrino flavours can be greatly enhanced. In fact, the $v_{\mathrm{e}}$ 's born inside the Sun have to cross a dense material in the first part of their path and the oscillation parameters are modified by the presence of the matter due to the different weak interaction for $v_{e}$ and $v_{\mu}$ on electrons. The parameters $\theta$ and $\Delta m^{2}$ that give the oscillation probability are modified in $\theta_{c}$ and $\Delta m_{\mathrm{c}}^{2}$ by this effect according to the formulae

$$
\left\{\begin{array}{l}
\sin ^{2} 2 \theta_{\mathrm{c}}=\sin ^{2} 2 \theta /\left(1-2 x \cos 2 \theta+x^{2}\right) \\
\Delta m_{\mathrm{c}}^{2}=\Delta m^{2}\left(1-2 x \cos 2 \theta+x^{2}\right)
\end{array}\right.
$$

where

$$
x=\lambda / \lambda_{0}, \quad \lambda=4 \pi E / \Delta m^{2}, \quad \lambda_{0}=2 \pi /\left(\sqrt{2} G N_{\mathrm{e}}\right)
$$

with $G$ being the weak-coupling constant and $N_{\mathrm{e}}$ the number of electrons per unit volume. Note that for a given $\Delta m^{2}, \lambda$ depends on neutrino energy, while $\lambda_{0}$ depends on the density of the matter traversed. One obtains a resonant condition when $\lambda=\lambda_{0} \cos 2 \theta$.

It is clear that the neutrino oscillation will change the energy spectra on the Earth of the $v_{e}$ coming from the Sun in a way depending on the values of the oscillation parameters and the matter density profile in the Sun. An extensive computation of the effect of neutrino oscillations on the solar-v flux can be found in ref. ${ }^{(7)}$. We summarize here the main conclusions:

a) If the Davis result is due to the neutrino oscillation, the dashed region in the $\left(\Delta m^{2}, \sin ^{2} 2 \theta\right)$-plane shown in fig. 2 gives rise to the measured discrepancy between the expected and the measured neutrino spectra.

b) For $\Delta m^{2} \sim 10^{-4}\left(\mathrm{eV}^{2}\right)$ the $v_{\mathrm{e}}$ flux is practically unaffected in the lowenergy region $(E<3 \mathrm{MeV})$ by the oscillation mechanism. Therefore, an experiment like GALLEX $\left.{ }^{8}\right)$, which detects solar neutrinos via the reaction

$$
v_{\mathrm{e}}+\mathrm{Ga} \rightarrow \mathrm{Ge}+\mathrm{e}^{-}
$$

with an energy threshold of $233 \mathrm{keV}$, will measure the flux expected in the standard solar model as though there were no oscillation effects. Therefore, if

${ }^{(6)}$ S. P. Mikheyev and A. YU. Smirnov: Nuovo Cimento C, 9, 17 (1986); L. WolfEnsteIn: Phys. Rev. D, 34, 969 (1336).

(7) S. P. Rosen and J. M. Gelb: Phys. Rev. D, 34, 969 (1986).

(8) Heidelberg, Karlsruhe, Munich, SaClay, Paris, Nice, Milano, Roma, REHOVOT: GALLEX proposal 1984. 
A POSSIBLE ACCELERATOR EXPERIMENT ETC.

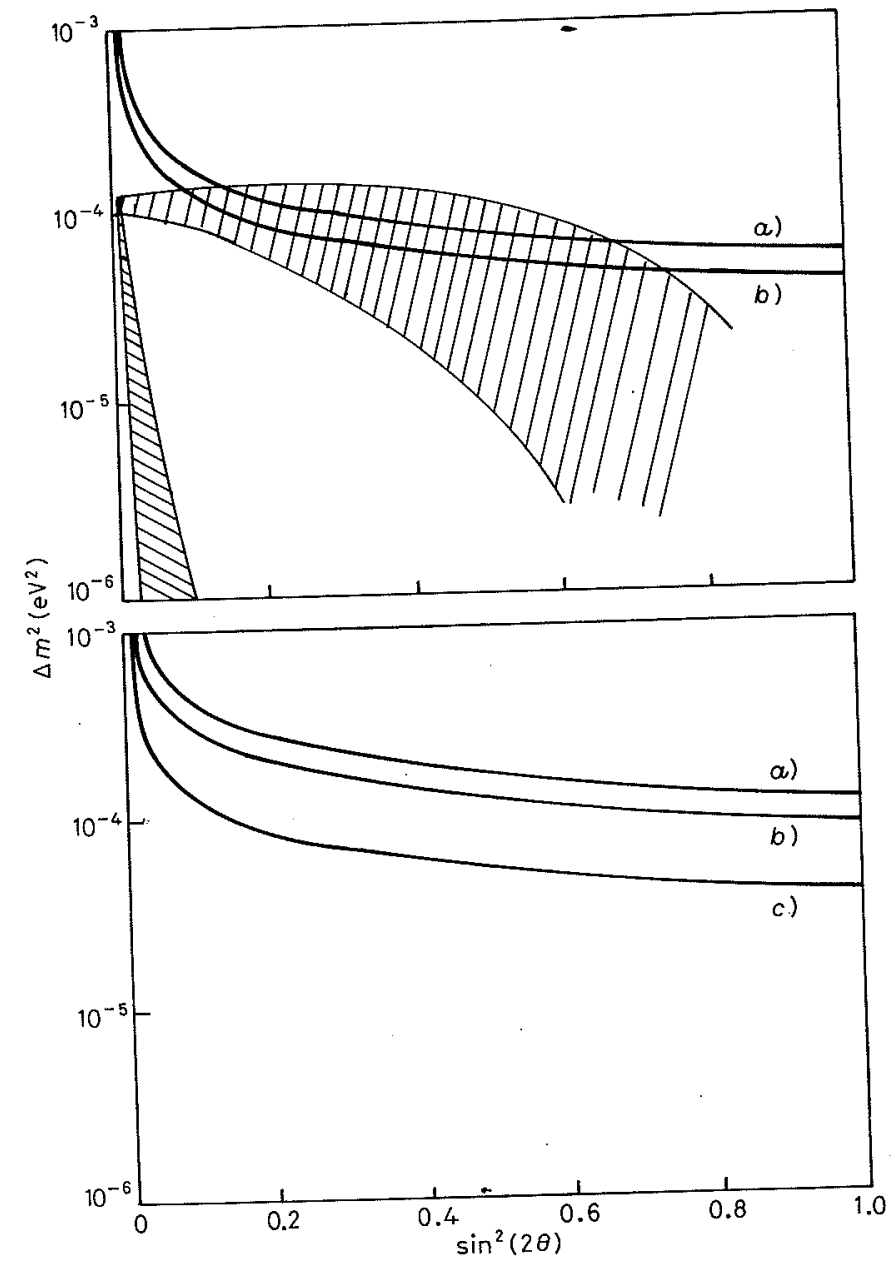

Fig. 2 . - Correlated limits on the oscillation parameters (90\% c.l.) for configuration $A(700$ $\mathrm{km})$ (bottom) and $B(1500 \mathrm{~km})(\mathrm{top})$ for this experiment. The limits correspond to $\left.10^{3}(a)\right)$, $\left.2 \cdot 10^{3}(b)\right)$ and $\left.10^{4}(c)\right) \vee$ interactions. The shaded area shows the region corresponding to the solution of the Davis experiment.

GALLEX measures a $v_{e}$ flux consistent with the standard solar model, it will become crucial to have an independent measurement, such as that we are discussing, to distinguish between neutrino oscillations and other effects to explain the Davis result.

\section{3. - Experimental scheme.}

The scheme of the experiment is shown in fig. 3 . The $\pi$ and $\mathrm{K}$ produced by the interaction of the $30 \mathrm{GeV}$ protons are focussed by means of a magnetic horn and 


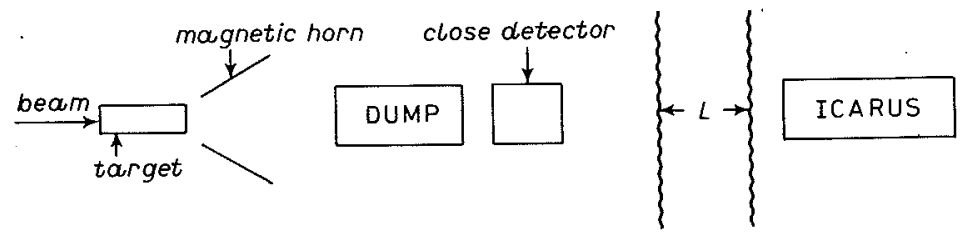

Fig. 3. - Scheme of the experimental lay out.

are allowed to decay over a $\sim 200 \mathrm{~m}$ long tunnel directed toward the Gran Sasso laboratory. At a distance of $\sim 1 \mathrm{~km}$ from the target a detector is placed, similar to ICARUS but with a much smaller mass $(\sim 10 \mathrm{t})$. This detector, referred to in the following as «close detector», which will identify the neutrino interactions and will measure the energy distribution as well as the $v_{e}$ content of the beam, is to be compared with the «far detector» ICARUS placed at a distance $L$. The combination of the intense neutrino flux with the large fiducial mass $\left(\sim 5 \cdot 10^{3} \mathrm{t}\right)$ of the far detector allows one to collect in a few months about thousand interactions at a distance of $\sim 10^{3} \mathrm{~km}$. This large value of $L$ that has never been reached by accelerator experiments will strongly enhance the sensitivity to low values of $\Delta m^{2}$ according to (4). In the proposed experiment, one starts with an almost pure $v_{y}$ beam, coming from pion and kaon décay, with $a_{s}$ small $v_{e}$ contamination mainly coming from $\mathrm{K} \rightarrow \pi \mathrm{e} v_{\mathrm{e}}$ and $\mu \rightarrow \mathrm{e} v_{\mathrm{e}} v_{\mu}$ decays. The possible $v_{e}-v_{\mu}$ oscillation will appear as an increase of the $v_{e} / v_{\mu}$ ratio in the «far» with respect to the «close» detector.

31. Beam. - In order to have an estimate of the neutrino flux, we have considered the recent experimental results $\left(^{9}\right)$ of the Brookhaven wide-band neutrino beam. In this case a $28 \mathrm{GeV}$ proton beam hits a sapphire target, $6.4 \mathrm{~mm}$ in diameter and $45 \mathrm{~cm}$ long. Secondary particles of selected sign are focussed in a toroidal magnetic field produced by a system of two horns, shown schematically in fig. 4. The result is a nearly parallel beam in the decay region. The Brookhaven $v_{\mu}-v_{\mathrm{e}}$ fluxes were measured from quasi-elastic neutrino events in a detector, placed at about $200 \mathrm{~m}$ from the target, and compared with the predictions given by the Monte Carlo program NUBEAM $\left({ }^{10}\right)$. The program generates particles according to a production spectrum and traces the selected

(9) L. A. Ahrens, S. H. Aronson, P. L. Connoluy, B. G. Gibbard, M. J. Murtagh, S. Terada, D. H. White, J. L. Callas, D. Cutts, M. Diwan, J. S. Hoftun, R. E. Lanou, T. Shinkawa, Y. Kurihara, K. Amako, S. Kabe, Y. Nagashima, Y. Suzuki, S. Tatsumi, Y. Yamaguchi, K. Abe, E. W. Beier, D. C. Doughty, L. S. DuRkin, S. M. Heagy, M. Hurley, A. K. Mann, F. M. Newcomer, H. H. Williams, T. York, D. HeIdIN, M. D. MARK and E. STERN: Phys. Rev. D, 34, 75 (1986).

$\left({ }^{10}\right)$ C. VISSER: NUBEAM, a neutrino-beam simulator, HYDRA application library, CERN (1979). 
A POSSIBLE ACCELERATOR EXPERIMENT ETC.

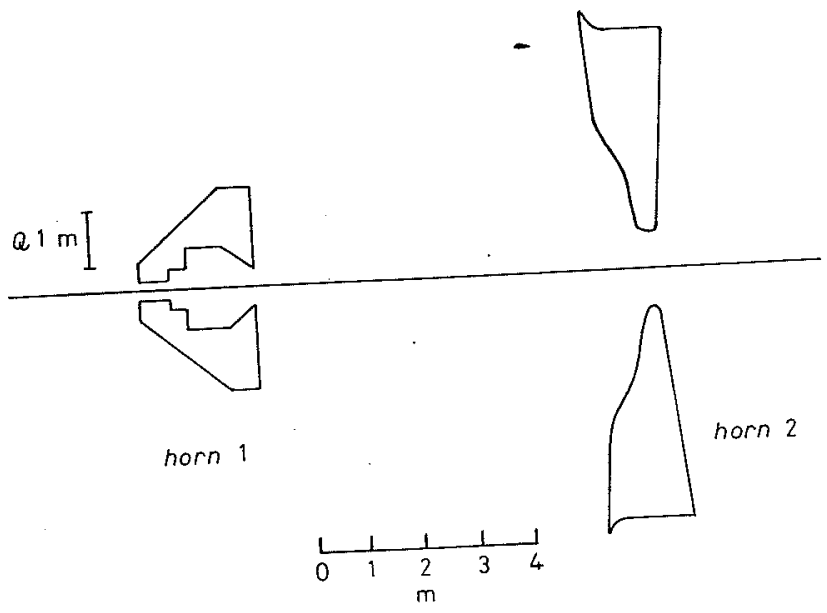

Fig. 4. - Scheme of the magnetic-horn system taken from ref. $\left(^{(}\right)$.

particles through the beam elements, until decay occurs or the particle is absorbed. The results are shown in fig. 5. As one can see, the agreement between MC and data is excellent. Therefore, we estimate the expected neutrino flux using the same program and the same horn scheme. The spectra obtained for $v_{y}$ and $v_{e}$ are presented in fig. 6. The $v_{e}$ contamination in the $v_{p}$ beam is $\sim 0.2 \%$. The $v_{\mu}$ spectrum has a maximum around $1.5 \mathrm{GeV}$ and is sharply decreasing for lower energy. However, we would like to point out that by

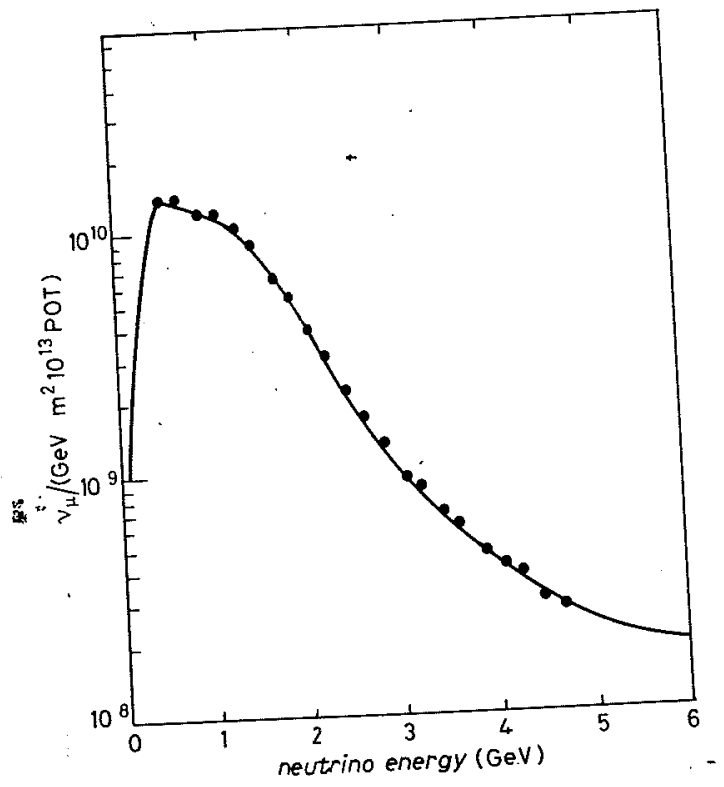

Fig. 5. - The AGS measured $v_{\sharp}$ flux (dots) together with the MC predictions (conţinuous line). 


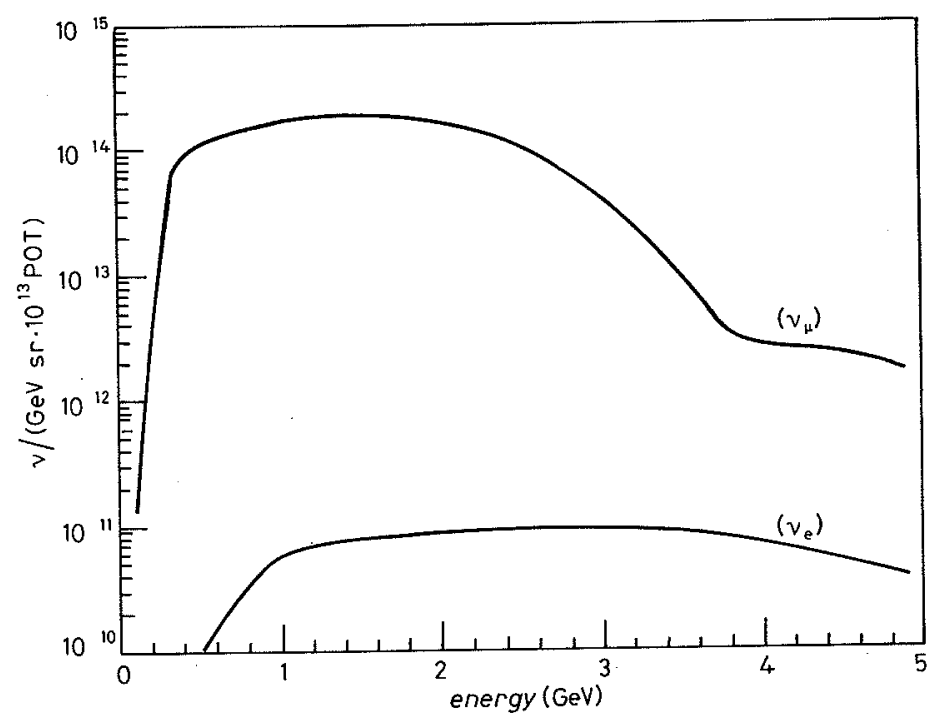

Fig. 6. - MC prediction for the expected $\nu_{\mu}$ and $\nu_{\mathrm{e}}$ spectra.

changing the shape and the material of target and the focussing device one can obtain a beam with a higher $v_{\mu}$ flux in the low-energy region $(E<2.0 \mathrm{GeV})$, where, according to (4), the sensitivity to low values of $\Delta m^{2}$ is enhanced. For the present estimate we use the fluxes given in fig. 6 , that are based on experimental data.

32. Detector. - A detailed description of ICARUS is given in ref. $\left(^{3}\right)$. We 品。 recall here only its main characteristics. It is (fig. 7) a large-volume $\left(4000 \mathrm{~m}^{3}\right)$

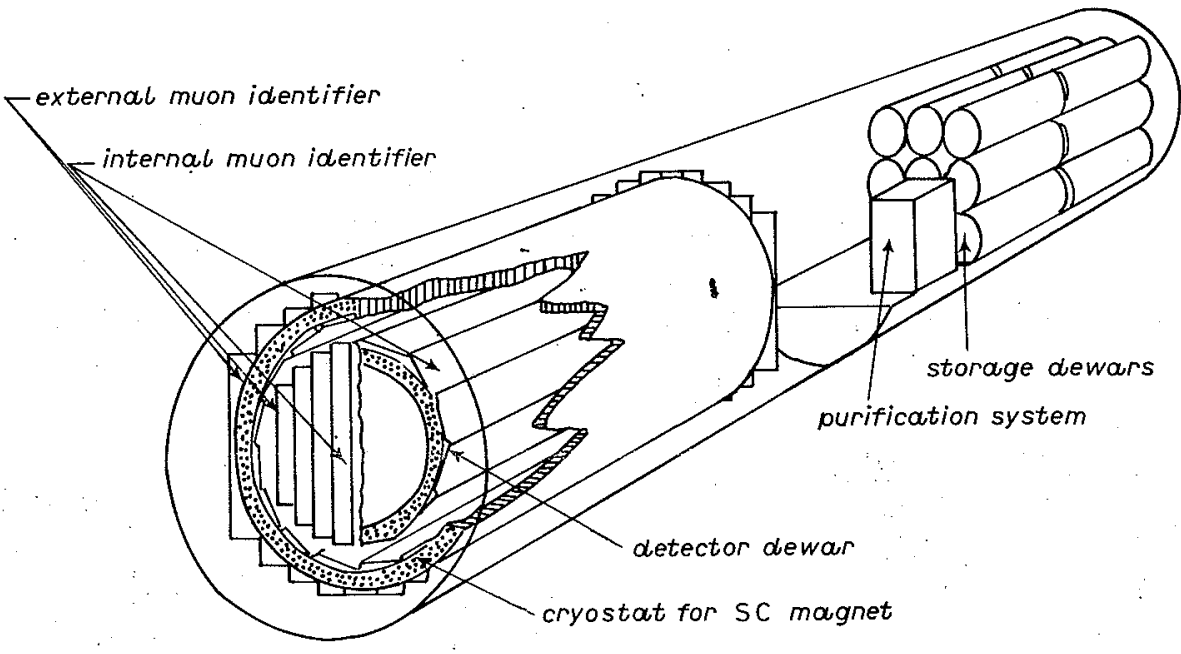

Fig. 7. - Artist's view of the ICARUS .detector. 
liquid-argon homogeneous detector, fully and continuously radiation sensitive. The ionization-induced electrons are collected over an about $1.5 \mathrm{~m}$ long path and are sensed by three planes of wires, which record the three-dimensional image of events occurring inside the chamber. The quality of the events is practically that of a bubble chamber; therefore, the identification of the electron produced in the reaction $v_{e}+\mathrm{N} \rightarrow \mathrm{e}^{-}+\mathrm{X}$ is unambiguous in the energy range of interest $(100 \mathrm{MeV} \div 10 \mathrm{GeV})$. Moreover, ICARUS gives an accurate measurement of the energy losses and it is surrounded by a solenoid with a $0.5 \mathrm{~T}$ magnetic field that permits momentum reconstruction in each interaction.

$3 \cdot 3$. Counting rate and sensitivity. - We compute the sensitivity for the $v$ oscillation parameters in the $\left(\Delta m^{2}, \sin ^{2} 2 \theta\right)$-plane using the fluxes given in fig. 6 , for two distances $L$ between the accelerator and the Gran Sasso laboratory:

a) $L=700 \mathrm{~km}$ (CERN-Gran Sasso), referred to as configuration $A$,

b) $L=1500 \mathrm{~km}$, referred to as configuration $B$.

With a proton beam of $6.3 \cdot 10^{14} \mathrm{p} / \mathrm{s}$ and with a $5 \cdot 10^{3} \mathrm{t}$ ICARUS fiducial mass, using the neutrino cross-section reported in fig. 8 , thousand interactions correspond to $\sim 60 \mathrm{~d}$ in configuration $A$ and $\sim 280 \mathrm{~d}$ in configuration $B(100 \%$ beam availability). The limits $(90 \%$ c.l. $)$ in the $\left(\Delta m^{2}, \sin ^{2} 2 \theta\right)$-plane corresponding to $10^{3}$ and $2 \cdot 10^{3}$ interactions are shown in fig. 2 for both configurations. These limits are computed taking into account the expected $v_{e}$ contamination in the apparatus with a statistical technique based on the maximum-likelihood ratio described, for example, in ref. $\left.{ }^{11}\right)$. In fig. 2 is also shown the limit one obtains in configuration $A$ with $10^{4}$ interactions that may be collected in about three years of effective running time (50\% overall efficiency). The sensitivity in this latter case is similar to that achievable in the same time with $2 \cdot 10^{3}$ events in configuration $B$. For this large number of events, however, we believe that the systematic uncertainties may affect the results.

We remark that a similar sensitivity can be reached with an underground detector looking at the atmospheric neutrinos $\left({ }^{12}\right)$. Neutrinos produced in the atmosphere by $\pi$ and $\mathrm{K}$ decay can travel across the Earth and reach the detector

(ii) C. Angelini, A. מatpostolakis, A. Baldini, M. Baldo-Ceolin, L. Bertanza, V. Flaminio, W. J. Fry, H. Huzita, P. Ioannou, S. Katsanevas, C. Kourkoumelis, J. Koutentakis, M. Loreti, R. Lovelles, G. Miari, R. Pazzi, P. Pramantiotis, M. Procario; G. Puglierin, D. D. Reeder, L. K. Resvanis, B. Saitta and M. VASSILIOU: Phys. Lett. B, 179, 307 (1986).

( $\left.{ }^{12}\right)$ J. LoSecco, R. M. Bionta, G. Blewitt, C. G. Bratton, D. Casper, P. Chrysicopoulou, R. Claus, B. G. Cortez, S. Errede, G. W. Foster, W. Gajewski, K. S. Ganzer, M. Goldhaber, T. J. Haines, T. W. Jones, D. KielczewsKa, W. R Kropp, J. G. Learned, E. Lehmann, H. S. Park, F. Reines, J. Schultz, S. Seidel, E. Shumard, D. Sinclair, H. W. Sobel, J. L. Stones, L. Sulak, R. Svoboda, J. C. VANDER Velde and C. West: Phys. Rev. Lett., 54, 2299 (1985). 


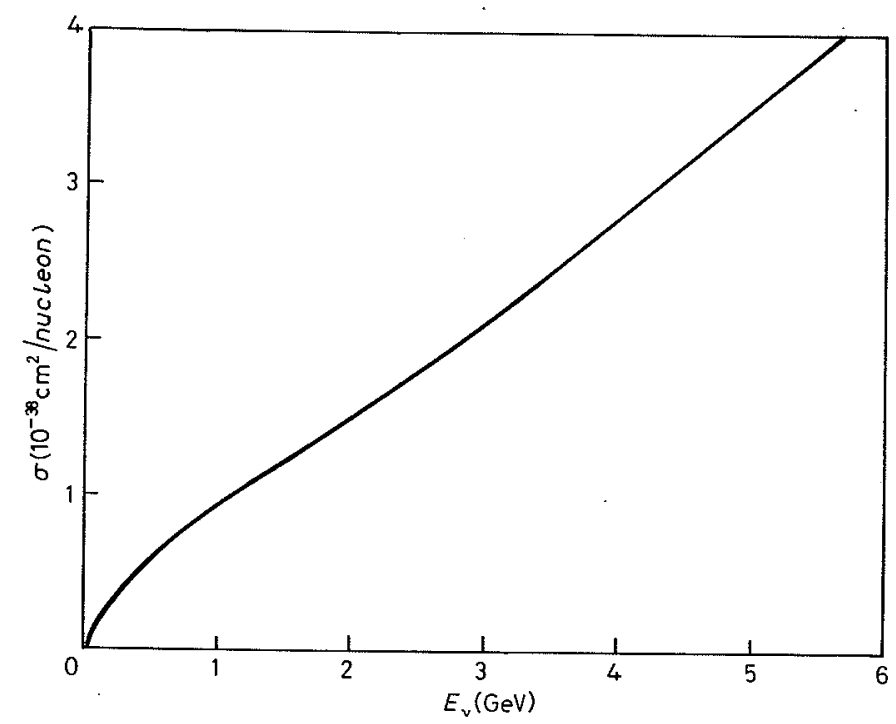

Fig. 8. $-v_{e} A r \rightarrow e^{-} X$ cross-section.

located on the opposite side. The distance that the neutrinos travel is a function of their zenith angle. An oscillation effect will thus appear as a dependence of the ratio $v_{e} / v_{\mu}$ on the zenith angle. Since the average atmospheric-neutrino energy is $\sim 1$. GeV (about the same as that of the beam we are considering) and the typical distance traversed is of the order of the diameter of the Earth $\left(10^{4} \mathrm{~km}\right)$, these experiments are sensitive to $\Delta m^{2} \sim\left(10^{-4} \div 10^{-5}\right) \mathrm{eV}^{2}$. In practice this kind of experiment is complicated by a) poor knowledge of the expected flux; $b$ ) simultaneous presence of $\left.v_{e}, v_{\mu}, \bar{v}_{e}, \bar{v}_{\mu} ; c\right)$ poor statistics; $d$ ) matter effects in the Earth. Therefore, the results are always obtained under several assumptions. The reliability of an experiment of the kind we are proposing is incomparably better.

3.4. Background - Since ICARUS is placed in an underground laboratory, the only background comes from the interactions of the atmospheric $v_{e}$ 's. The number of interactions due to these atmospheric neutrinos is known experimentally and it amounts to $\sim 10^{3}$ events/y in the $5 \cdot 10^{3} \mathrm{t}$ of ICARUS. About $20 \%$ of these events are expected to be $v_{\mathrm{e}}$. To reject this background one can use:

a) The time information; the EHF $\vee$ beam will have a short spill, with a repetition rate of $12.5 \mathrm{~Hz}$. Requiring a time coincidence, the resolution time is dominated by ICARUS and is $\sim 10^{-4} \mathrm{~s}$. Therefore, the rejection factor is $10^{-3}$.

b) Angular distribution of electrons; the neutrinos coming from the accelerator are obviously all parallel. The electrons produced in the reaction $v_{e} \mathrm{~N} \rightarrow \mathrm{e}^{-} \mathrm{X}$ are peaked in the forward direction. Taking into account nuclear 
effects, one obtains the angular distribution for the electrons shown in fig. 9 . This permits us to apply an angular cut with respect to the $v$ beam to discriminate against the isotropic background with a rejection factor of $\sim 0.1$.

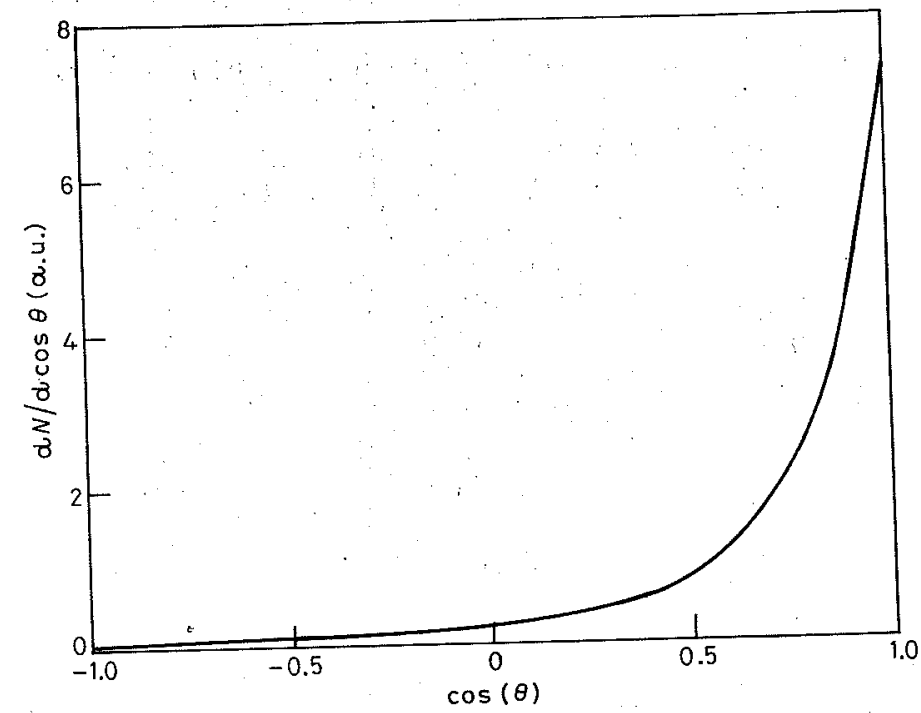

Fig. 9. - MC prediction for electron angular distribution in the reaction $v_{e} A r \rightarrow e^{-} X$.

Combining the time and angular information the cosmic-ray background becomes completely negligible.

Another background comes from the misidentification of a $v_{\psi}$ interaction as $v_{\mathrm{e}}$. This background has been estimated to be small compared to the expected $v_{e}$ contamination in the beam.

It has to be remarked that the uncertainties, in the estimate of the contamination from the beam and from the background, are removed by comparing the far and the close detector and, therefore, do not introduce systematic effects but at most spoil slightly the sensitivity.

\section{4. - Conclusions."}

The proposed experiment improves the limits, obtained with reactors or accelerators $\left.{ }^{(13}\right)$, in $\Delta m^{2}$ by two or three orders of magnitude.

The comparison with solar-neutrino experiments turns out to be of particular interest if the accelerator is placed at $-1500 \mathrm{~km}$ from the Gran Sasso

$\left.{ }^{13}\right)$ For a recent review of neutrino oscillation experiments see V. FLAMINIo and $B$. SAITTA: Riv. Nuovo Cimento, 10, No. 8 (1987) 


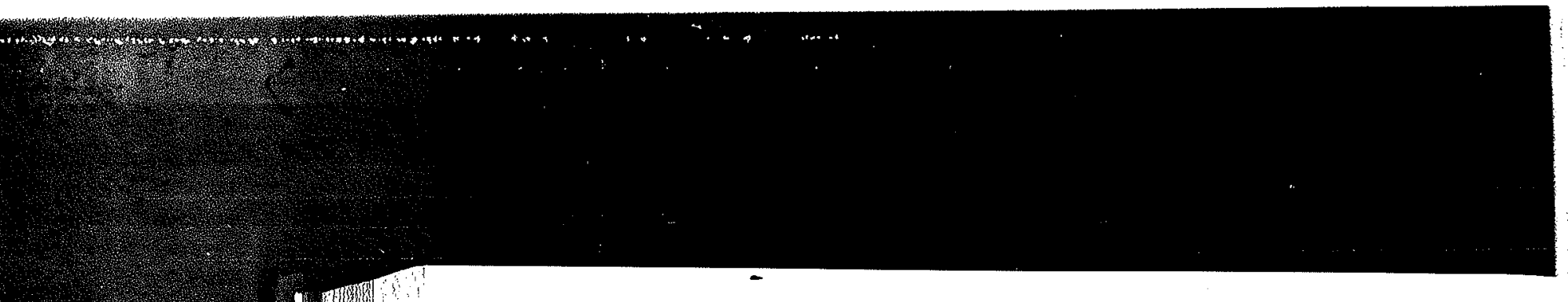

A. CAPONE, H. WAChSMUTh, V. PALladino, M. DE VINCENZI, ETC.

(configuration $B$ ). In this case, in about three years of effective running time (50\% overall efficiency), one can reach a sensitivity to values $\Delta m^{2} \sim 10^{-4}$, $\sin ^{2} 2 \theta \geq 0.15$. This region of values of the oscillation parameters is of crucial importance in the solar-model problem. A similar sensitivity can be reached for a distance $L=700 \mathrm{~km}$ by collecting $\sim 10^{4}$ interactions ( $3 \mathrm{y}$ of running time); however, the significance of this experiment is minor because of the systematic uncertainties.

It has also to be remarked that the information obtained from solar-neutrino experiments concerns the oscillation of $\nu_{e}$ into any other flavour $\left(v_{\mu}, v_{-}\right)$, while the proposed experiment directly measures $P\left(v_{e} \rightarrow v_{\mu}\right)$ and, therefore, gives independent information.

$$
* * *
$$

It is a pleasure to thank Prof. M. Conversi and Dr. F. Massa for enlightening discussions and useful advices.

\section{RIASSUNTO}

Si mostra la possibilità di usare un fascio intenso di neutrini gerferati da un acceleratore assieme ad un rivelatore sotterraneo per studiare le oscillazioni de neutrini. In particolare si discutono le configurazioni nelle quali la sensibilità nei parametri di oscillazione è confrontabile con quella degli esperimenti sui neutrini solari.

\section{Возможный эксперимент на ускорителе для решения проблемы солнечных нейтрино.}

Резюме (*). - Мы показываем возможность использования интенсивного потока нейтрино от ускорителя вместе с подземным детектором для исследования осцилляций нейтрино. В частности, мы обсуждаем конфигурации, при которых чувствительность к параметрам осцилляций будет соизмерима с чувствительностью экспериментов с солнечными нейтрино.

(*) Переведено редакцией. 